\title{
Tidal stripping as a test of satellite quenching in redMaPPer clusters
}

\author{
Yuedong Fang, ${ }^{1 \star}$ Joseph Clampitt, ${ }^{1}$ Neal Dalal, ${ }^{2}$ Bhuvnesh Jain, ${ }^{1}$ Eduardo Rozo, ${ }^{3}$ \\ John Moustakas ${ }^{4}$ and Eli Rykoff ${ }^{5}$ \\ ${ }^{1}$ Department of Physics and Astronomy, Center for Particle Cosmology, University of Pennsylvania, 209 S. 33rd St, Philadelphia, PA 19104, USA \\ ${ }^{2}$ Department of Astronomy, University of Illinois at Urbana-Champaign, 1002 West Green St, Urbana, IL 61801, USA \\ ${ }^{3}$ Department of Physics, University of Arizona, 1118 E 4th St, Tucson, AZ 85721, USA \\ ${ }^{4}$ Department of Physics and Astronomy, Siena College, Loudonville, NY 12211, USA \\ ${ }^{5}$ SLAC National Accelerator Laboratory, Menlo Park, CA 94025, USA
}

Accepted 2016 August 18. Received 2016 August 15; in original form 2016 May 24

\begin{abstract}
When dark matter haloes are accreted by massive host clusters, strong gravitational tidal forces begin stripping mass from the accreted subhaloes. This stripping eventually removes all mass beyond a subhalo's tidal radius, with unbound mass remaining in the vicinity of the satellite for at most a dynamical time $t_{\text {dyn }}$. The $N$-body subhalo study of Chamberlain et al. verified this picture and pointed out a useful observational consequence: correlations between subhaloes beyond the tidal radius are sensitive to the infall time, $t_{\text {infall }}$, of the subhalo on to its host. We perform this correlation using $\sim 160000$ red satellite galaxies in Sloan Digital Sky Survey redMaPPer clusters and find evidence that subhalo correlations do persist well beyond the tidal radius, suggesting that many of the observed satellites fell into their current host less than a dynamical time ago, $t_{\text {infall }}<t_{\text {dyn }}$. Combined with estimated dynamical times $t_{\text {dyn }} \sim 3-5 \mathrm{Gyr}$ and SED fitting results for the time at which satellites stopped forming stars, $t_{\text {quench }} \sim 6 \mathrm{Gyr}$, we infer that for a significant fraction of the satellites, star formation quenched before those satellites entered their current hosts. The result holds for red satellites over a large range of cluster-centric distances $0.1-0.6 \mathrm{Mpc} h^{-1}$. We discuss the implications of this result for models of galaxy formation.
\end{abstract}

Key words: galaxies: clusters: general-galaxies: formation.

\section{INTRODUCTION}

Clusters of galaxies - composed of tens to hundreds of satellite galaxies orbiting within a dark matter halo - are the most massive virialized objects in the Universe. Their average dark matter profiles and halo masses have been measured in great detail (e.g. Mandelbaum et al. 2006, 2010; Johnston et al. 2007; Mandelbaum, Seljak \& Hirata 2008; Rozo et al. 2009; Sheldon et al. 2009). Recent work has measured finer features of the mass distribution within and around cluster haloes. The splashback radius predicted by Diemer \& Kravtsov (2014) and Adhikari, Dalal \& Chamberlain (2014) was recently detected by More et al. (2016). Halo assembly bias (Sheth \& Tormen 2004; Gao, Springel \& White 2005; Wechsler et al. 2006; Dalal et al. 2008) has also been detected by the same group (Miyatake et al. 2016; More et al. 2016). Evidence for cluster ellipticity from weak lensing has been measured on small samples of $\sim 20$ clusters (Oguri et al. 2010; Donahue et al. 2016) as well as large samples of several thousand clusters (Evans \& Bridle
2009; Clampitt \& Jain 2016). Similarly, filaments between individual pairs of massive clusters (Dietrich et al. 2012; Jauzac et al. 2012) and thousands of group and cluster pairs (Clampitt et al. 2016) have recently been detected using weak gravitational lensing, supplementing similar measurements with the cluster galaxy distribution (Zhang et al. 2013).

While this work has been accomplished with existing data such as the Sloan Digital Sky Survey ${ }^{1}$ (SDSS), the next generation of surveys including the Dark Energy Survey ${ }^{2}$ (DES; Melchior et al. 2015; Nord et al. 2016; Rykoff et al. 2016), Hyper Suprime-Cam ${ }^{3}$ (HSC; Miyazaki et al. 2015) and the Kilo Degree Survey ${ }^{4}$ (KiDS; Viola et al. 2015) have also begun producing results on galaxy clusters. Within a few years, these new surveys will provide much larger cluster samples than were previously available.

Compared to their massive host clusters, the properties of cluster subhaloes are less well studied. Subhaloes were once isolated dark

\footnotetext{
${ }^{1}$ http://www.sdss.org

2 http://www.darkenergysurvey.org

${ }^{3}$ http://www.naoj.org/Projects/HSC/

${ }^{4}$ http://kids.strw.leidenuniv.nl
} 
matter haloes, each with their own central galaxy, before gravitational forces pulled them inside much larger neighbouring haloes. Many such accreted haloes are tidally destroyed and become indistinguishable as distinct entities, but subhaloes are the survivors that persist as satellites of the larger cluster halo. Since the lensing and clustering signals of subhaloes are dwarfed by their massive host clusters, it is more difficult to study their detailed mass distributions. Recent progress in determining weak lensing masses of subhaloes has been made by Li et al. (2016) using Canada-FranceHawaii Telescope (CFHT) Stripe-82 data (Erben et al. 2013) and by Sifón et al. (2015) with KiDS data (Kuijken et al. 2015). While simulation studies of subhaloes predict that tidal forces from the host cluster will strip mass from the subhalo outskirts (Hayashi et al. 2003; Gao et al. 2004), the recent lensing studies were not able to identify a tidal radius beyond which mass was stripped. However, the Li et al. (2016) measurements hinted that subhaloes closer to the cluster centre - where tidal forces are strongest - may be less massive, which would provide indirect evidence for tidal stripping.

Given the insufficient signal to noise (S/N) of weak lensing, correlations between subhaloes within the same host provide a promising alternative way to measure tidal stripping, as demonstrated by Chamberlain et al. (2015) using subhaloes in $N$-body simulations. Cohn (2012) and Cohn \& White (2014) had shown the existence of such correlations and discussed a variety of consequences in physical and velocity space. Chamberlain et al. (2015) pointed out that subhalo-subhalo correlations can be used to address an important open question in galaxy formation: did the star formation of satellites galaxies end upon accretion? The striking colour difference between galaxies in cluster and field environments - satellites are mostly 'red and dead' while field galaxies are mostly blue and actively forming stars - has prompted a search for the mechanisms within clusters that end star formation in satellites. These include accretion shocks (Balogh, Navarro \& Morris 2000; Dekel \& Birnboim 2006), strangulation (Larson, Tinsley \& Caldwell 1980), ram-pressure stripping (Gunn \& Gott 1972; Abadi, Moore \& Bower 1999), and the effect of many high-speed encounters with other satellites (Farouki \& Shapiro 1981; Moore et al. 1996) - all of which act to either forcibly remove gas from the subhalo or prevent it from cooling enough to form stars.

It is possible to reproduce many observed statistics of quiescent galaxies using models relying upon quenching of star formation caused by intracluster processes. For example, Wetzel et al. (2013) used a galaxy group catalogue from SDSS Data Release 7, combined with the quiescent fractions from COSMOS survey, and a cosmological $\mathrm{N}$-body simulation to study the star formation histories of satellite galaxies at $z \approx 0$. They found that satellite quenching is consistent with the statistics of quiescent galaxies if quenching is a 'delayed-then-rapid' process: the satellites remain actively star forming for 2-4 Gyr after their first infall, unaffected by the host halo, after which the quenching occurs rapidly with a star formation rate (SFR) e-folding time $<0.8 \mathrm{Gyr}$.

Alternatively, it is also possible that other processes besides satellite quenching may be dominant in determining quiescent fractions in clusters. For example, the age-matching model of Hearin \& Watson (2013) can reproduce many measurements of galaxy luminosity, colour, clustering, and weak lensing (Hearin et al. 2014; Watson et al. 2015) without relying on satellite quenching processes. By placing the reddest galaxies in the oldest dark matter haloes which tend to be the ones that formed in denser environments - the Hearin \& Watson (2013) model naturally reproduces the large red fraction seen in observations.
However, recently Zu \& Mandelbaum (2016) showed that the original age-matching model of Hearin \& Watson (2013) is in tension with measurements of the halo mass of isolated blue galaxies (Mandelbaum et al. 2016). $\mathrm{Zu} \&$ Mandelbaum (2016) study two quenching models and show that a model in which halo mass alone determines quenching fits the SDSS measurements ( $\mathrm{Zu} \&$ Mandelbaum 2015; Mandelbaum et al. 2016) better than a hybrid model which depends on stellar mass and host halo mass (for satellites). While both models do well at fitting red galaxy clustering and lensing, the halo quenching model is much better at modelling the halo mass for massive blue central galaxies. Zu \& Mandelbaum (2016) show that the colour dependence of the $\left\langle M_{h} \mid M_{*}\right\rangle$ relation in the publicly available mock galaxy catalogues of Hearin et al. (2014) is inconsistent with SDSS measurements, since this mock tends to place blue and red centrals of similar $M_{*}$ in haloes of similar mass, which is heavily disfavoured by the data. It is unclear whether this conclusion can be generalized to the broader class of age-matching models, or if it is specific to the original construction of Hearin \& Watson (2013), since Campbell, Padmanabhan \& van den Bosch (2016) argue that a slightly modified parametrization of age matching fits the measurements of $\mathrm{Zu} \&$ Mandelbaum (2016).

The detection of the splashback feature by More et al. (2016) provides additional insight into quenching processes in clusters. More et al. (2016) detect the splashback feature in both red and blue galaxies. This shows that galaxies can complete at least one full orbit within their host clusters while remaining unquenched (i.e. blue). Given that orbital times in the outskirts of haloes can be a large fraction of the Hubble time (e.g. $6 \mathrm{Gyr}$ ), this result may pose a challenge for the model of Wetzel et al. (2013). More et al (2016) also find that the red fraction of cluster galaxies exhibits a pronounced feature at the splashback radius $r_{\mathrm{sp}}$. While the naive interpretation of this sharp feature at the splashback radius is that crossing $r_{\mathrm{sp}}$ modifies galaxy colours (i.e. host-quenching), similar behaviour also naturally arises in age-matching models without host-quenching. In those models, the sharp feature in the red fraction is a consequence of the sharp transition at the splashback radius from the 2-halo region to the 1-halo region.

In light of these conflicting models, we carry out the subhalosubhalo clustering measurements proposed in Chamberlain et al (2015) in order to provide model-independent constraints on the relationship between subhalo infall and quenching times. Alternative models of galaxy formation may then be distinguished based on their predictions for the fraction of quiescent satellites that quenched upon accretion, rather than while isolated. We assume a flat universe and $\Omega_{\mathrm{m}}=0.3$. In Section 2, we describe our SDSS data samples. In Section 3, we describe our method of the measurement. In Section 4, we describe measurement results of subhalo correlations and compare infall, dynamical, and quenching time-scales for red SDSS satellites. In Section 5, we discuss possible systematics as well as implications of our measurements for models of galaxy formation.

\section{DATA}

For our subhalo sample, we use members of SDSS redMaPPer clusters (Rykoff et al. 2014; Rozo et al. 2015a). We use clusters with redshift $0.15<z<0.41$ and more than 10 member galaxies, i.e. we require the richness $\lambda>10$ (note that the public catalogue only goes down to $\lambda=20$ ). We also require the clusters to have a high redMaPPer centring probability, Pcen $>0.8$. These cuts reduce the number of clusters to 11800 . In addition to the cuts on cluster properties, we only use satellites with a membership probability 
Pmem $>0.8$, ensuring that our satellite sample is pure. Rozo et al. (2015a) has shown that the photometric cluster selection done by redMaPPer is effectively as good as a spectroscopic selection for those satellites with a high membership probability. We will be interested in studying the satellite clustering as a function of distance from the centre of the cluster, $r_{\mathrm{c}}$, so for this purpose we define three bins $r_{\mathrm{c}}=0.1-0.3 \mathrm{Mpc} h^{-1}, r_{\mathrm{c}}=0.3-0.6 \mathrm{Mpc} h^{-1}$, and $r_{\mathrm{c}}=$ $0.6-0.9 \mathrm{Mpc} h^{-1}$. The range in $r_{\mathrm{c}}$ for each bin was chosen to obtain sufficient $\mathrm{S} / \mathrm{N}$ in all three bins: each bin of increasing $r_{\mathrm{c}}$ has 110144 , 42742 , and 6728 galaxies.

We cross-correlate these subhaloes with the redMaGiC galaxy catalogue (Rozo et al. 2016). We use redMaGiC because many of these bright red galaxies are members of the redMaPPer clusters and may compose infalling groups with the redMaPPer members we study. We do not simply use the redMaPPer satellites because redMaPPer imposes a spatial selection around each cluster, rejecting all galaxies outside a radius $R_{\lambda}=1 \mathrm{Mpc} h^{-1}(\lambda / 100)^{0.2}$. This might introduce edge effects in our measurement so we use the spatially uniform redMaGiC sample for cross-correlations (see Section 4 for details). The redMaGiC algorithm selects only red galaxies with good photometric redshifts with a median bias $z_{\text {spec }}-z_{\text {photo }}$ of 0.005 and scatter $\sigma_{z} /(1+z)$ of 0.017 . When performing crosscorrelations, we select only redMaGiC galaxies within 0.02 of the cluster redshift.

For each redMaPPer member, we use SED fits to the broadband photometry to determine the age or time since the onset of star formation. The prior on star formation history is taken to be delayed tau models (Maraston et al. 2010), which produce a continuous but exponentially declining star formation history. We are most interested in when the subhalo quenched or stopped forming stars. Due to the exponential decline, it is not a bad approximation to take this measure of age as the quenching time, $t_{\text {quench }} \equiv$ $\left(\int \mathrm{d} t t \operatorname{SFR}(t)\right) / \int \mathrm{d} t \operatorname{SFR}(t)$, where we have used the SFR-weighted age. The initial mass function is taken from Salpeter (1955) over the range $0.1-100 \mathrm{M}_{\odot}$. See appendix A of Moustakas et al. (2013) for more details on the SED modelling. In Section 5.2, we discuss how this definition of $t_{\text {quench }}$ connects with definitions used in galaxy formation models.

\section{METHOD}

Chamberlain et al. (2015) used simulations to split subhaloes between those that were recently accreted and those accreted more than one dynamical time ago. The dynamical time was estimated as the period for a circular orbit at radius equal to the distance from satellite to its central galaxy. The results are shown in their fig. 2: the oldest subset showed no significant correlation at large radii. In contrast, the subhaloes which infell recently did show large-scale correlations. This means the measurement of large-scale correlations can be used to determine when subhaloes fell into their hosts. We have also tested that we recover similar results as Chamberlain et al. (2015), but using a larger simulation. These results are shown in Appendix A.

We seek to detect these large-scale correlations in data by crosscorrelating subhaloes (redMaPPer members) with the redMaGiC sample by counting the number of redMaGiC galaxies in annuli centred on each redMaPPer subhalo. However, since subhaloes are by definition always part of a larger halo, the raw number counts measured around subhaloes will be dominated by the host. In order to remove this host signal, we follow the method of Pastor Mira et al. (2011) and Chamberlain et al. (2015), subtracting the counts around the subhalo's 'mirror' point reflected about the cluster centre. Since

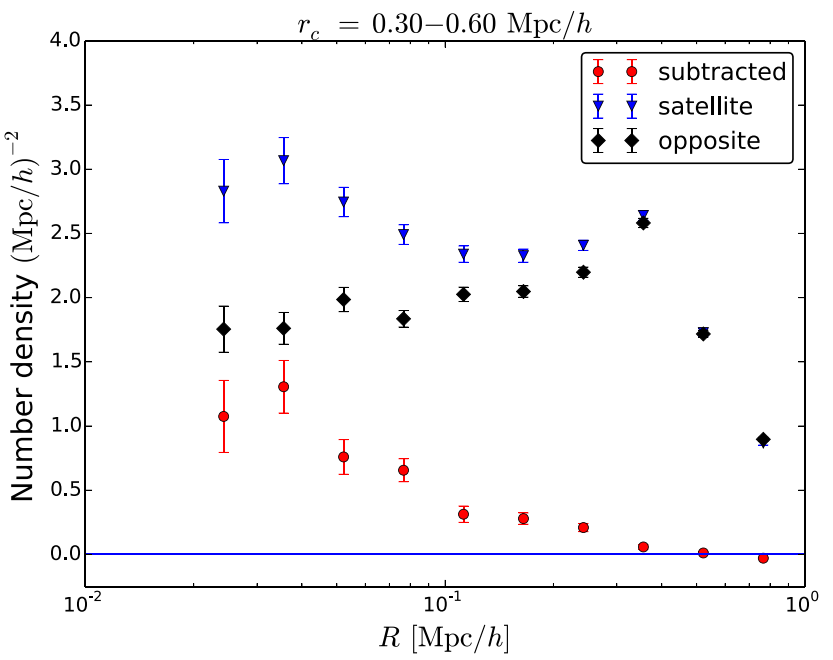

Figure 1. Number density of redMaGiC galaxies around redMaPPer members (blue points) and around their opposite positions relative to the cluster centre (black points). The difference (red points) isolates the subhalo clustering signal.

this point is at the same distance $r_{\mathrm{c}}$ from the cluster centre, if the host cluster profile is spherically symmetric it will have the same contribution from the host. This method is illustrated in Fig. 1, which shows the counts around the redMaPPer satellite and the opposite point. Both are dominated by the miscentred host signal, but after subtraction the subhalo clustering signal is clearly visible at small scales and falls smoothly to zero at large scales.

This subtracted signal is our estimator used in all remaining plots: we count redMaGiC galaxies in annuli centred on each redMaPPer subhalo, subtract the counts of redMaGiC galaxies around each mirror point, and divide by the number of subhaloes. For all measurements, we divide the SDSS data into 200 spatially uniform patches and estimate a jackknife covariance by removing each patch in turn (Norberg et al. 2009). All figures show the diagonal $1 \sigma$ jackknife errors while our $\mathrm{S} / \mathrm{N}$ estimates incorporate the full jackknife covariance.

Note that we measure correlations in 2D, projected bins of clustercentric distance $r_{\mathrm{c}}$. This is in contrast to fig. 2 of Chamberlain et al. (2015), which shows 3D correlations. In Section 5.1, we argue that the qualitative conclusions we draw from the 2D correlations should be similar to those based on 3D correlations. Moreover in future work, we intend to present correlations in deprojected 3D clustercentric distance bins to compare more directly with Chamberlain et al. (2015).

\section{RESULTS}

\subsection{Correlations beyond the tidal radius}

In Fig. 2, we show the results for three cluster-centric radius bins, $0.1<r_{\mathrm{c}}<0.3,0.3<r_{\mathrm{c}}<0.6$, and $0.6<r_{\mathrm{c}}<0.9 \mathrm{Mpc} h^{-1}$. The correlations are clearly detected in each case, and fall as $\sim 1 / R$ with increasing scale. Note there is an increase in correlations for larger $r_{\mathrm{c}}$ bins which may be due partly to the positive correlation of $r_{\mathrm{c}}$ with stellar mass (see Fig. 8). We also tested measurements in bins of $r_{\mathrm{c}} / R_{200}$, where $R_{200}$ is the host's virial radius. These results were qualitatively similar. In order to interpret the measurements of Fig. 2, we next estimate the subhaloes' tidal radius, beyond which unbound material can be stripped by tidal forces from the host. 


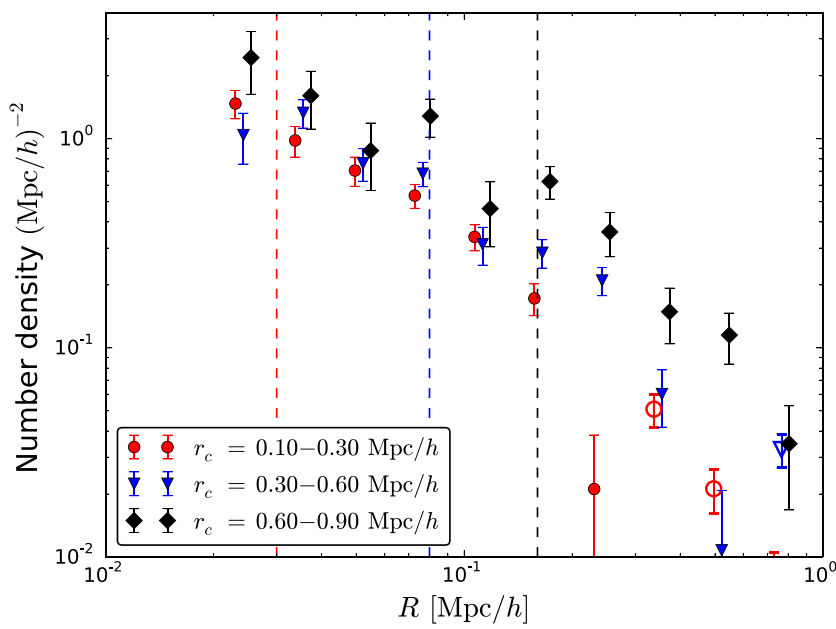

Figure 2. Projected number density of redMaGiC galaxies stacked around subhaloes, for all three bins of cluster-centric distance $r_{\mathrm{c}}$. Note that the data points have been shifted slightly along the $x$-axis for clarity. Above $\sim 0.1 \mathrm{Mpc} h^{-1}$, there is a clear trend of increasing correlations for larger cluster-centric distance. Vertical lines show the estimated tidal radius for each subhalo sample: all measurements show significant correlations with unbound material beyond the tidal radius.

Measuring correlations well beyond the tidal radius would indicate that at least some of the subhaloes were recently accreted, since otherwise unbound material would have been stripped. The 'Hill' radius, which can be used to estimate the tidal radius, is roughly the radius where $\rho_{\text {subhalo }} \sim \rho_{\text {host }}$ (Chamberlain et al. 2015), where the dynamical time for particles orbiting the subhalo becomes comparable to the dynamical time for the subhalo to orbit its host. To obtain $\rho_{\text {subhalo, }}$, we use the results of Li et al. (2016), which measured lensing masses for a subset of our subhaloes, those which overlap with the CFHT Stripe- 82 Survey. Li et al. (2016) found best-fitting subhalo masses $10^{11.3}, 10^{12.0}$, and $10^{12.5} \mathrm{M}_{\odot} h^{-1}$ for increasing distance from the cluster, using the same three $r_{\mathrm{c}}$ bins as our measurements. In Fig. 3, we show NFW density profiles (Navarro, Frenk \& White 1997) for the three bins using these masses.

To obtain the average local host density at the satellite locations, we use the mass-richness relation of Simet et al. (2016):

$M_{200}=10^{14.344} \mathrm{M}_{\odot} h^{-1} \times(\lambda / 40)^{1.33}$.

We then estimate $\rho_{\text {host }}(r)$ using this host mass and an NFW profile with the mass-concentration relation of Duffy et al. (2008), and plot the result in Fig. 3. Here, $r$ is the 3D cluster-centric distance. By assuming the host halo is spherically symmetric, and the distribution of satellites follows an NFW form, $r$ can be estimated by

$r\left(r_{\mathrm{c}}\right)=\frac{\int_{0}^{\sqrt{R_{200}{ }^{2}-r_{\mathrm{c}}^{2}}} \rho_{\mathrm{NFW}}\left(\sqrt{r_{\mathrm{c}}^{2}+x^{2}}\right) \times \sqrt{x^{2}+r_{\mathrm{c}}^{2}} \mathrm{~d} x}{\int_{0}^{\sqrt{R_{200^{2}-r_{\mathrm{c}}^{2}}}} \rho_{\mathrm{NFW}}\left(\sqrt{r_{\mathrm{c}}^{2}+x^{2}}\right) \mathrm{d} x}$.

The host and subhalo density profiles cross at $r_{\text {tidal }} \sim 0.03,0.07$, and $0.15 \mathrm{Mpc} h^{-1}$ for the three bins of increasing $r_{\mathrm{c}}$. A rough comparison to the simulation results of Chamberlain et al. (2015) shows that our results are comparable: for their $0.4 \mathrm{Mpc} h^{-1}<r_{\mathrm{c}}<$ $0.6 \mathrm{Mpc} h^{-1}$ bin, the local host density and subhalo density match at $\sim 0.045 \mathrm{Mpc}^{-1}$ (see fig. 1 of that work). This is slightly smaller but close to the tidal radius $\sim 0.07 \mathrm{Mpc} h^{-1}$ that we find for subhaloes between $0.3 \mathrm{Mpc} h^{-1}<r_{\mathrm{c}}<0.6 \mathrm{Mpc} h^{-1}$; exact agreement is not expected since we have not attempted a comparison with matched host halo masses.

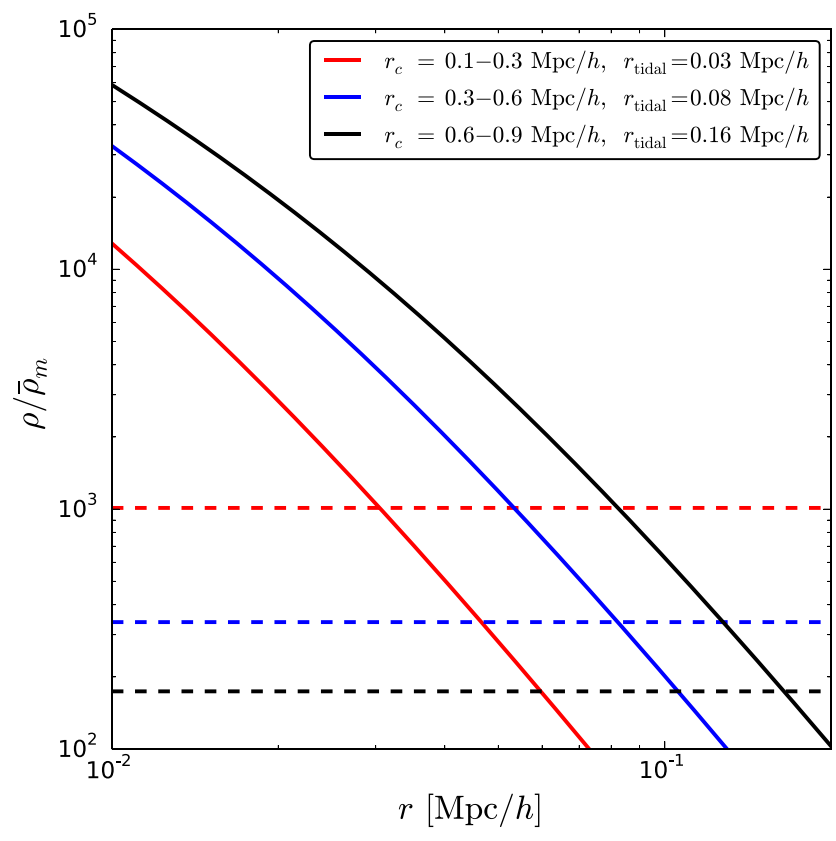

Figure 3. The subhalo profile (solid lines) and background host density (dashed lines) for each $r_{\mathrm{c}}$ bin. The estimated $r_{\text {tidal }}$ is the radius where the two density profiles cross.

Table 1. S/N of the correlations beyond the tidal radius shown in Fig. 2. The measurement is significant regardless of cluster-centric distance $r_{\mathrm{c}}$.

\begin{tabular}{|c|c|c|}
\hline \multirow[t]{2}{*}{$r_{\mathrm{c}}\left(\mathrm{Mpc} h^{-1}\right)$} & \multicolumn{2}{|c|}{$\mathrm{S} / \mathrm{N}$} \\
\hline & {$\left[r_{\text {tidal }}, r_{\mathrm{c}} / 2\right]$} & {$\left[r_{\text {tidal }}, r_{\mathrm{c}}\right]$} \\
\hline $0.1-0.3$ & 11.1 & 14.7 \\
\hline $0.3-0.6$ & 7.1 & 8.8 \\
\hline $0.6-0.9$ & 6.1 & 6.8 \\
\hline
\end{tabular}

Material at larger radii should be unbound, so we look for correlations beyond $r_{\text {tidal }} \sim 0.03,0.07$, and $0.15 \mathrm{Mpc}^{-1}$, for each of the three $r_{\mathrm{c}}$ bins. Comparing these scales to Fig. 2, we do see correlations well beyond $r_{\text {tidal }}$ in each case. We find the $\mathrm{S} / \mathrm{N}$ using points in the range $r_{\text {tidal }}$ to $r_{\mathrm{c}} / 2$ is $11.1,10.3$, and 6.1 corresponding to the three bins of increasing $r_{\mathrm{c}}$. Thus, all show significant detections. This implies that some of the subhaloes recently fell into their hosts and there has not been enough time for unbound material to be torn away, i.e. $t_{\text {infall }}<t_{\text {dyn }}$, where $t_{\text {infall }}$ is the infall time and $t_{\text {dyn }}$ is the dynamical time. In Table 1 , we also show the $\mathrm{S} / \mathrm{N}$ using a wider range of scales $r_{\text {tidal }}<R<r_{\mathrm{c}}$, but note that points close to $r_{\mathrm{c}}$ may be subject to several systematics discussed in Section 5.1. In the following section, we estimate the dynamical time and compare it to the quenching time-scale for the subhaloes.

\subsection{Dynamical and quenching time-scales}

We can estimate the dynamical time for each subhalo using its distance from the cluster centre $r$ and the host density profile. As in Section 4.1, we estimate the host density using the redMaPPer mass-richness relation and assume an NFW profile. The dynamical time is then

$t_{\mathrm{dyn}}=2 \pi \sqrt{r^{3} /(G M(<r))}$, 


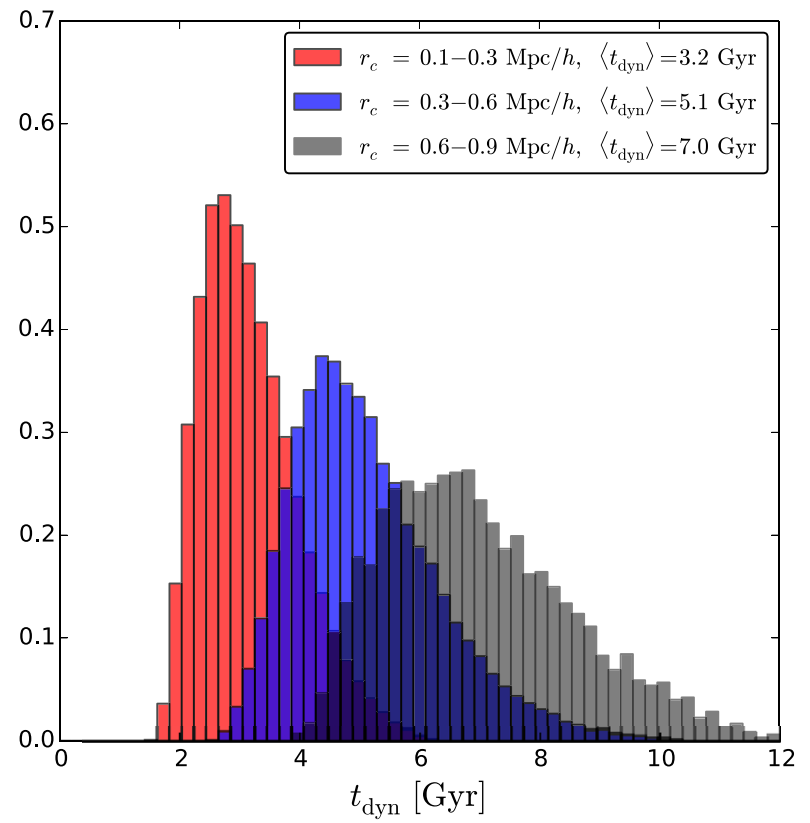

Figure 4. Distribution of dynamical times of the subhaloes for each $r_{\mathrm{c}}$ bin. The dynamical time is estimated using equation (3). Here, we use the redMaPPer relation and assume an NFW profile to estimate the host mass. It can be seen that the dynamical time is increasing with $r_{\mathrm{c}}$ with the mean value for the outer most bin $t_{\mathrm{dyn}} \sim 6 \mathrm{Gyr}$.

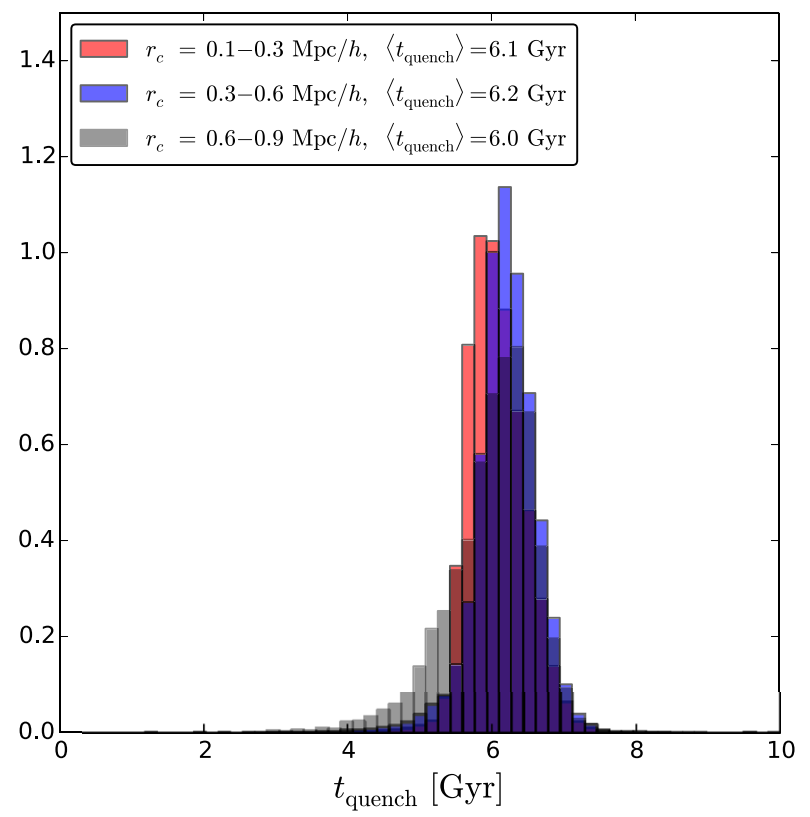

Figure 5. Quenching time since the redMaPPer subhaloes stopped forming stars. For all three $r_{\mathrm{c}}$ bins, the mean quenching time is $\sim 6$ Gyr. Thus, for the inner two $r_{\mathrm{c}}$ bins we have $t_{\mathrm{dyn}}<t_{\text {quench }}$.

where $M(<r)$ is the host mass contained within the 3D radius $r$, which is estimated by equation (2). In Fig. 4, we show histograms of the resulting dynamical times, for the three $r_{\mathrm{c}}$ bins. The mean is small for the innermost bin, $\sim 3 \mathrm{Gyr}$, increasing to $\sim 6 \mathrm{Gyr}$ for the galaxies farthest from the cluster centre. This is expected due to the smaller density at larger distances from the cluster centre.

We next compare these dynamical times to the quenching time determined from SED fits (see details in Section 2). In Fig. 5, we show the distribution of $t_{\text {quench }}$ using the mean of the posterior for each subhalo. The distribution peaks at $\sim 6 \mathrm{Gyr}$, a value greater than the dynamical times $\sim 3-4$ Gyr (see Fig. 4) for subhaloes with $r_{\mathrm{c}}<0.6 \mathrm{Mpc} h^{-1}$. Thus, many of our subhaloes have $t_{\text {infall }}<t_{\text {dyn }}$ $<t_{\text {quench }}$, implying that these galaxies ceased star formation long before being accreted by their hosts.

This would be inconsistent with models of star formation in which these subhaloes were actively forming stars up until the time they fell into their current hosts. In such models, infall into the cluster leads to stripping of the gas and quenching of star formation. Hence, such models require that $t_{\text {quench }}<t_{\text {infall }}$ for a large fraction of our subhaloes.

\subsection{Other tests}

As an additional test of the quenching scenario, we have redone the measurement using the older half of subhaloes in Fig. 5, most of which have $t_{\text {quench }}>6 \mathrm{Gyr}$. The results are shown in Fig. 6, and again we see strong correlations well beyond the tidal radius. For a more model-independent test of age, we split the subhaloes into halves based on colour, a proxy for age since the reddest, deadest subhaloes are the ones that ceased forming stars long ago. In Fig. 7, we show the correlations measured around the 'redder' subhaloes, defined as those which are redder than the redMaPPer red sequence model at that redshift. Again we see strong correlations beyond the tidal radius for all $r_{\mathrm{c}}$ bins. Thus, many of these subhaloes recently fell into their hosts, long after they had ceased forming stars.

We also tested the dependence of our results on the specific galaxy samples by measuring the autocorrelation of redMaPPer satellites. This gave similar results with correlations persisting well beyond the tidal radius. The redMaPPer satellites are dimmer than redMaGiC with a cut at $L>0.2 L_{*}$ instead of $L>0.5 L_{*}$. But redMaPPer also enforces a spatial selection around the cluster centre, which may bias the redMaPPer autocorrelation for the larger $r_{\mathrm{c}}$ bins. For this reason, we did not choose the autocorrelation for our fiducial measurements.

\section{DISCUSSION}

We have shown that redMaGiC galaxies are significantly correlated with redMaPPer satellites, on angular scales exceeding the estimated tidal radii for satellites at small cluster-centric distances. This result holds even when we subselect the reddest satellites, or those believed to have been quenched for the longest times. Our measurements can be compared with the simulation results of Chamberlain et al (2015), and suggest that a significant fraction of the massive satellites in our sample were quenched prior to infall into their host cluster haloes. Future studies with ongoing surveys using the clustering of satellite galaxies and their weak lensing signal can shed light on galaxy formation models and probe possible interactions of dark matter. Below, we discuss potential systematics in our measurements, and then discuss implications for satellite quenching models.

\subsection{Possible systematics}

First, we discuss possible systematics in our measurement of correlations beyond the tidal radius. Two effects which we have neglected will become increasingly important at projected distances $\sim r_{\mathrm{c}}$, i.e. near and beyond the cluster centre. The first is oversubtraction of the subhalo profile. When we measure correlations around the mirror point (see Section 3 and Fig. 1), there will be some positive 

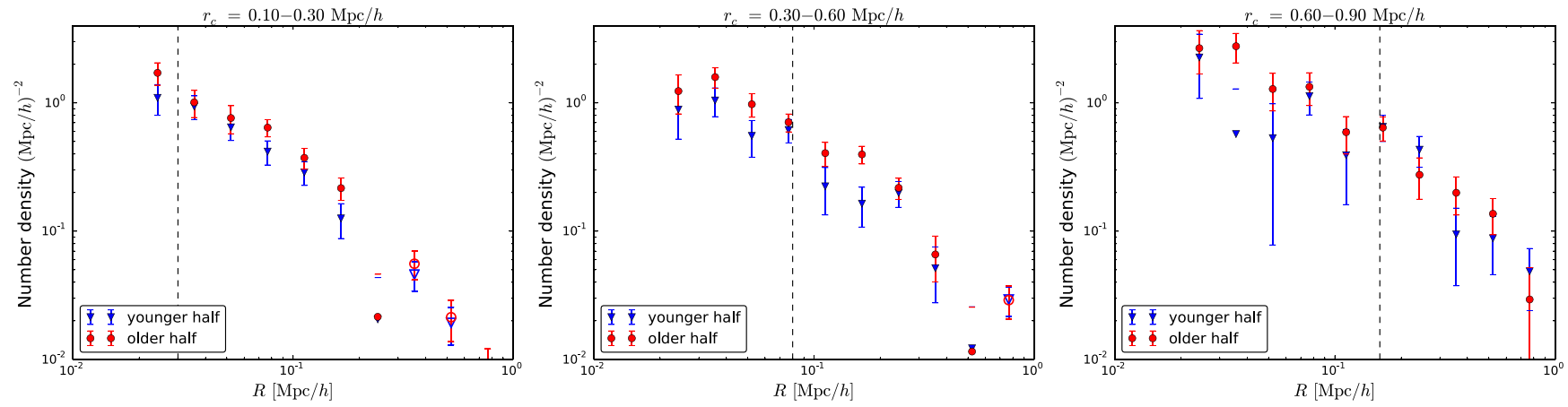

Figure 6. Comparison between correlations of older (red circles) and younger (blue triangles) subhaloes, as determined from SED fit results for $t_{\text {quench. }}$ Each panel shows results for subhaloes at different distances $r_{\mathrm{c}}$ from the host cluster. Subhaloes with $r_{\mathrm{c}}<0.6 \mathrm{Mpc} h^{-1}$ show correlations well beyond the tidal radius (vertical dashed line). These long-range correlations are present for both the young and old samples.
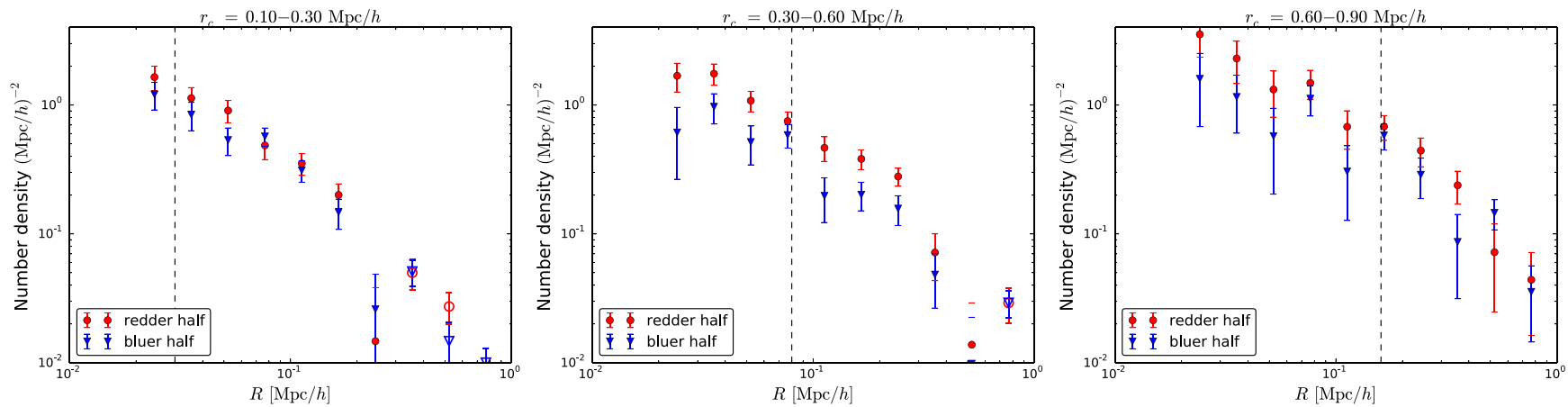

Figure 7. Same as Fig. 6 but showing subhaloes split by colour: those redder (red circles) and bluer (blue triangle) than the red sequence model. Since redder galaxies have been quiescent for longer, these results are similar to the $t_{\text {quench }}$ split but less dependent on detailed SED modelling.

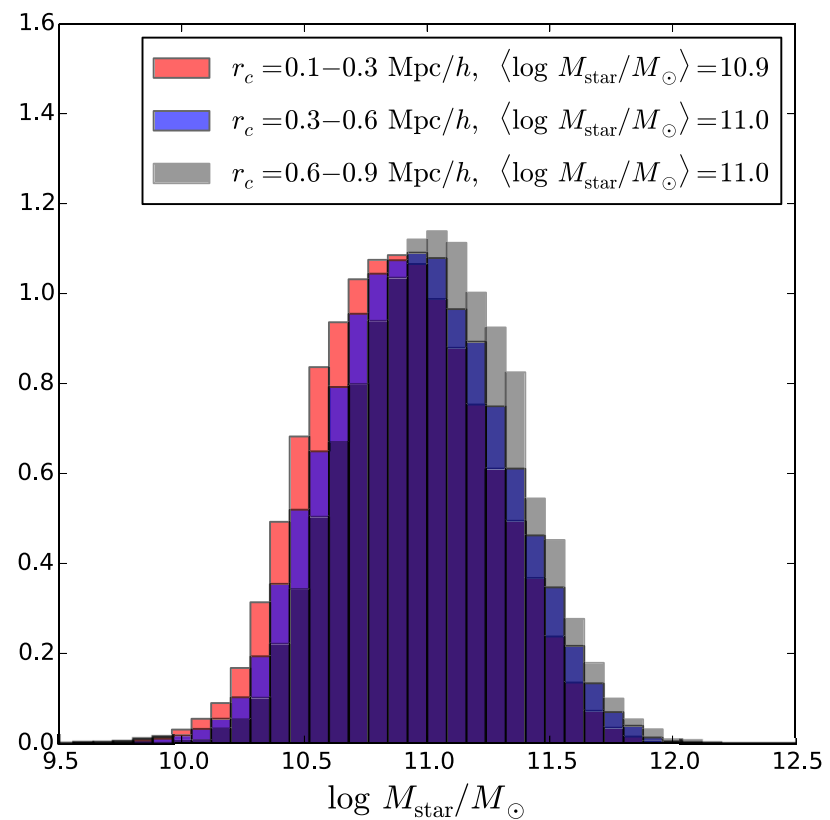

Figure 8. Normalized stellar mass distributions for the three cluster distance bins.

contribution from the subhalo, the magnitude of which will become important at scales $R \sim 2 r_{\mathrm{c}}$ when the annulus $R$ contains the subhalo. Note that this systematics contributes with negative amplitude due to the subtraction and so does not weaken our case for the detection of subhalo correlations beyond the tidal radius.
A second effect could result from systematic cluster miscentering. If the true cluster centre is on average closer to the subhalo than redMaPPer's most probable centre, then the subtraction of the mirror-point signal will imperfectly remove correlations due to the host cluster. This would contribute most strongly at larger scales $R \sim r_{\mathrm{c}}$ : as seen in Fig. 1, the cluster signal as measured around the opposite point is roughly flat within $R \sim r_{\mathrm{c}} / 2$, then begins rising to peak at $R \sim r_{\mathrm{c}}$ before decreasing again. Thus, residuals of the subtracted cluster signal could cause a roughly constant systematic below $r_{\mathrm{c}} / 2$ and increase in magnitude at larger scales. We found no evidence of such a trend in our measurements. Note that we only use clusters with a centre that is both (i) the most likely centre for that cluster and (ii) truly looks like a central: these are galaxies which are bright, red, and near the centre of the spatial distribution of cluster members. See Rykoff et al. (2014) for more information on the redMaGiC centring filter and Rozo \& Rykoff (2014) for further tests of redMaPPer centring, including comparison to X-ray centres for a subset of clusters.

Due to imprecise line-of-sight information on galaxy positions, some fraction of the redMaPPer members may lie outside the halo. Assuming this interloper component has a constant spatial density, it will be most important at large $r_{\mathrm{c}}$ since the profile of actual cluster galaxies drops with distance from the cluster centre. According to Rozo et al. (2015a), the fraction of such interlopers is estimated to be 6 per cent averaged over the whole cluster. If interlopers were responsible for much of the signal we detect, the measured angular correlation for neighbours around redMaPPer galaxies at small $r_{\mathrm{c}}$ would be $\sim 16 \times$ smaller than the analogous correlation function at $r_{\mathrm{c}} \gg r_{\mathrm{vir}}$. We do not find this, so conclude that interlopers are not a significant factor in our analysis. 

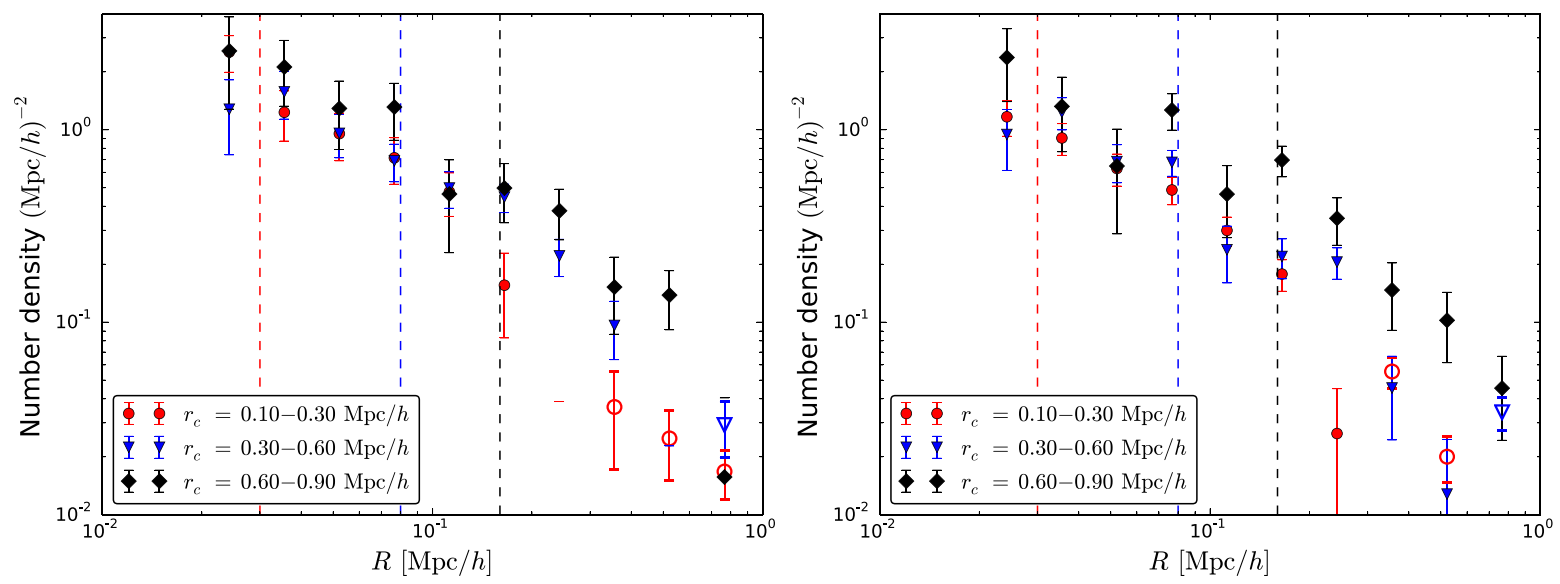

Figure 9. Projected number density as in Fig. 2, but split by satellite stellar mass: higher (left-hand panel) and lower (right-hand panel) than $10^{11} \mathrm{M}_{\odot} h^{-1}$.

The previous paragraph discussed the effect of projections of distant interlopers, next we discuss the effect of two types of less catastrophic projections. First, we measure correlations with redMaGiC galaxies in 2D-projected distance (i.e. the $x$-axis of Fig. 2 is a projected quantity). Chamberlain et al. (2015) showed that results for subhalo-subhalo correlations in 3D and in projection are qualitatively similar. For example, in their figs 3 and 4, they show that 3D and projected correlations show the same features between different cluster-centric distance bins. Thus, we do not worry further about this type of projection effect. Secondly, we use 2D cluster-centric bins $r_{\mathrm{c}}$, meaning that each $r_{\mathrm{c}}$ bin contains subhaloes over a range of larger 3D distances. In this paper, we have partially accounted for this effect by estimating the average 3D cluster-centric distance via equation (2), and using that mean 3D quantity to estimate $r_{\text {tidal }}$ and $t_{\mathrm{dyn}}$. In addition, we have carried out a deprojection of our angular correlations for the two lower $r_{\mathrm{c}}$ bins; preliminary results suggest that the long-range correlations persist. We will present a quantitative comparison of our deprojected correlations with galaxy formation models in a separate study.

\subsection{Implications for models of galaxy formation}

We have argued that the absence of tidal stripping favours isolated quenching of a significant fraction massive galaxies, prior to their infall into galaxy clusters. Here, we compare our results to host-quenched fractions predicted by more specific models of galaxy formation. The model of Wetzel et al. (2013) predicts a strong dependence of the host-quenched fraction with subhalo stellar mass and host halo mass. Our clusters have mass $\gtrsim 10^{14} \mathrm{M}_{\odot} h^{-1}$, comparable to the most massive cluster bin studied by Wetzel et al. (2013). In Fig. 8, we show our subhalo stellar mass distributions: all three bins have similar distributions with mean mass $\sim 10^{11} \mathrm{M}_{\odot}$, with a very slight shift to larger stellar mass for larger $r_{\mathrm{c}}$. For this host mass and subhalo stellar mass, fig. 10 of Wetzel et al. (2013) indicates that 55 per cent of quiescent satellites quenched as satellites (40 per cent in the current host and 15 per cent as satellites of a prior host; 45 per cent quenched while isolated). This estimate appears to be consistent with our findings that a large fraction of redMaPPer satellites quenched prior to entering their current hosts. It will be interesting, therefore, to perform the same measurement for satellites with lower stellar masses, where the Wetzel et al. (2013) model predicts nearly $\sim 100$ per cent quenched as satellites. Such measurements should be possible with ongoing imaging surveys like DES, HSC, or DECALS, or future surveys like LSST. Within the range of satellite stellar masses in our data (see Fig. 8), we have split the sample into two with median stellar masses of about $4.6 \times 10^{10}$ and $1.7 \times 10^{11} \mathrm{M}_{\odot} h^{-1}$. The results are shown in Fig. 9; it is evident that deeper imaging data that obtains cluster satellites with lower stellar mass is needed to explore possible trends.

Another reason it will be interesting to pursue similar measurements for lower mass galaxies is that the Wetzel et al. (2013) model requires a delay between the time when star-forming satellites fell into their first host and the time when their star formation began to exponentially decay. Their fig. 8 shows that this delay time is $\sim 2.5$ Gyr for our subhaloes. Since our SED fits imply that $t_{\text {quench }}$ $\sim 6$ Gyr, the Wetzel et al. (2013) model would predict that first infall occurred $\sim 8.5$ Gyr ago (i.e. at $z>1$ ) in order for satellites to be host-quenched. We see no evidence for such high redshifts of infall. For satellites with $M_{\star} \sim 10^{11} \mathrm{M}_{\odot}$, a large fraction are predicted to quench as centrals, so our measurements are not inconsistent with the model's predictions. However, if we find similar results for satellites with $M_{\star} \sim 10^{10} \mathrm{M}_{\odot}$, it will be difficult to reconcile that behaviour with host quenching models like the Wetzel et al. (2013) model in which nearly 100 per cent of objects quenched as satellites. One possible solution to this problem is if a significant fraction of the red satellites quenched as satellites of previous (smaller) hosts, rather than their current hosts. For the host-satellite properties that match our data, Wetzel et al. (2013) estimate that only $\sim 15$ per cent of such galaxies quenched as satellites in hosts different than their current hosts, and it is unclear whether this small fraction would be sufficient to account for the correlations observed in our sample. We intend to address some of these open questions in a future study that includes the use of deprojected, $3 \mathrm{D}$ correlations of satellites for quantitative comparisons with theoretical models.

\section{ACKNOWLEDGEMENTS}

We would like to thank the referee for helpful comments and suggestions. We also thank Gary Bernstein, Daniel Gruen, Eric Baxter, Juliana Kwan, Mike Jarvis, Vinicius Miranda, Vishal Kasliwal, Tamas Norbert Varga, Ravi Sheth, and Risa Wechsler for helpful discussions. YF and BJ are partially supported by the US Department of Energy grant DE-SC0007901. ND thanks the Institute for Advanced Study for hospitality during the course of this work. 


\section{$1914 \quad$ Y. Fang et al.}

\section{REFERENCES}

Abadi M. G., Moore B., Bower R. G., 1999, MNRAS, 308, 947

Adhikari S., Dalal N., Chamberlain R. T., 2014, J. Cosmol. Astropart. Phys., 11,019

Balogh M. L., Navarro J. F., Morris S. L., 2000, ApJ, 540, 113

Campbell D., Padmanabhan N., van den Bosch F. C., 2016, in SnowPAC 2016: The Galaxy-Halo Connection. Available at: http://www.physics.utah.edu/snowpac/talks/campbell.pdf

Chamberlain R. T., Dalal N., Hearin A., Ricker P., 2015, MNRAS, 451, 1496

Clampitt J., Jain B., 2016, MNRAS, 457, 4135

Clampitt J., Miyatake H., Jain B., Takada M., 2016, MNRAS, 457, 2391

Cohn J. D., 2012, MNRAS, 419, 1017

Cohn J. D., White M., 2014, MNRAS, 440, 1712

Dalal N., White M., Bond J. R., Shirokov A., 2008, ApJ, 687, 12

Dekel A., Birnboim Y., 2006, MNRAS, 368, 2

Diemer B., Kravtsov A. V., 2014, ApJ, 789, 1

Dietrich J. P., Werner N., Clowe D., Finoguenov A., Kitching T., Miller L., Simionescu A., 2012, Nature, 487, 202

Donahue M. et al., 2016, ApJ, 819, 36

Duffy A. R., Schaye J., Kay S. T., Dalla Vecchia C., 2008, MNRAS, 390, L64

Erben T. et al., 2013, MNRAS, 433, 2545

Evans A. K. D., Bridle S., 2009, ApJ, 695, 1446

Farouki R., Shapiro S. L., 1981, ApJ, 243, 32

Gao L., White S. D. M., Jenkins A., Stoehr F., Springel V., 2004, MNRAS, 355,819

Gao L., Springel V., White S. D. M., 2005, MNRAS, 363, L66

Gunn J. E., Gott J. R., III, 1972, ApJ, 176, 1

Hayashi E., Navarro J. F., Taylor J. E., Stadel J., Quinn T., 2003, ApJ, 584, 541

Hearin A. P., Watson D. F., 2013, MNRAS, 435, 1313

Hearin A. P., Watson D. F., Becker M. R., Reyes R., Berlind A. A., Zentner A. R., 2014, MNRAS, 444, 729

Jauzac M. et al., 2012, MNRAS, 426, 3369

Johnston D. E. et al., 2007, preprint (arXiv:0709.1159)

Klypin A., Yepes G., Gottlober S., Prada F., Hess S., 2016, MNRAS, 457, 4340

Kuijken K. et al., 2015, MNRAS, 454, 3500

Larson R. B., Tinsley B. M., Caldwell C. N., 1980, ApJ, 237, 692

Li R. et al., 2016, MNRAS, 458, 2573

Mandelbaum R., Seljak U., Cool R. J., Blanton M., Hirata C. M., Brinkmann J., 2006, MNRAS, 372, 758

Mandelbaum R., Seljak U., Hirata C. M., 2008, J. Cosmol. Astropart. Phys., 8,006

Mandelbaum R., Seljak U., Baldauf T., Smith R. E., 2010, MNRAS, 405, 2078

Mandelbaum R., Wang W., Zu Y., White S., Henriques B., More S., 2016, MNRAS, 457, 3200

Maraston C., Pforr J., Renzini A., Daddi E., Dickinson M., Cimatti A., Tonini C., 2010, MNRAS, 407, 830

Melchior P. et al., 2015, MNRAS, 449, 2219

Miyatake H., More S., Takada M., Spergel D. N., Mandelbaum R., Rykoff E. S., Rozo E., 2016, Phys. Rev. Lett., 116, 041301
Miyazaki S. et al., 2015, ApJ, 807, 22

Moore B., Katz N., Lake G., Dressler A., Oemler A., 1996, Nature, 379, 613

More S. et al., 2016, ApJ, 825, 39

Moustakas J., 2013, ApJ, 767, 50

Navarro J. F., Frenk C. S., White S. D. M., 1997, ApJ, 490, 493

Norberg P., Baugh C. M., Gaztañaga E., Croton D. J., 2009, MNRAS, 396, 19

Nord B. et al., 2016, ApJ, 827, 51

Oguri M., Takada M., Okabe N., Smith G. P., 2010, MNRAS, 405, 2215

Pastor Mira E., Hilbert S., Hartlap J., Schneider P., 2011, A\&A, 531, A169

Rozo E., Rykoff E. S., 2014, ApJ, 783, 80

Rozo E. et al., 2009, ApJ, 699, 768

Rozo E., Rykoff E. S., Becker M., Reddick R. M., Wechsler R. H., 2015, MNRAS, 453, 38

Rozo E. et al., 2016, MNRAS, 461, 1431

Rykoff E. S. et al., 2014, ApJ, 785, 104

Rykoff E. S. et al., 2016, ApJS, 224, 1

Salpeter E. E., 1955, ApJ, 121, 161

Sheldon E. S. et al., 2009, ApJ, 703, 2232

Sheth R. K., Tormen G., 2004, MNRAS, 350, 1385

Sifón C. et al., 2015, MNRAS, 454, 3938

Simet M., McClintock T., Mandelbaum R., Rozo E., Rykoff E., Sheldon E. Wechsler R. H., 2016, preprint (arXiv:1603.06953)

Viola M. et al., 2015, MNRAS, 452, 3529

Watson D. F. et al., 2015, MNRAS, 446, 651

Wechsler R. H., Zentner A. R., Bullock J. S., Kravtsov A. V., Allgood B., 2006, ApJ, 652, 71

Wetzel A. R., Tinker J. L., Conroy C., van den Bosch F. C., 2013, MNRAS, 432,336

Zhang Y., Dietrich J. P., McKay T. A., Sheldon E. S., Nguyen A. T. Q., 2013, ApJ, 773, 115

Zu Y., Mandelbaum R., 2015, MNRAS, 454, 1161

Zu Y., Mandelbaum R., 2016, MNRAS, 457, 4360

\section{APPENDIX A: SIMULATION}

We briefly show results from $N$-body simulations to demonstrate the effects of tidal stripping exhibited by subhaloes that have been in their host for more than a dynamical time. We use the MultiDark Planck 2 simulation (Klypin et al. 2016), available from the CosmoSim data base. ${ }^{5}$ In Fig. A1, we plot correlations from subhaloes within hosts of comparable mass to Chamberlain et al. (2015). The left-hand panel shows subhaloes accreted long ago, with accretion redshifts range chosen to be longer than a dynamical time for all the bins. As expected, these subhaloes have been in their hosts sufficiently long that long-range correlations have completely decayed. The right-hand panel shows subhaloes accreted recently: less than a dynamical time ago. These profiles are consistent with no tidal stripping.

${ }^{5}$ https://www.cosmosim.org 

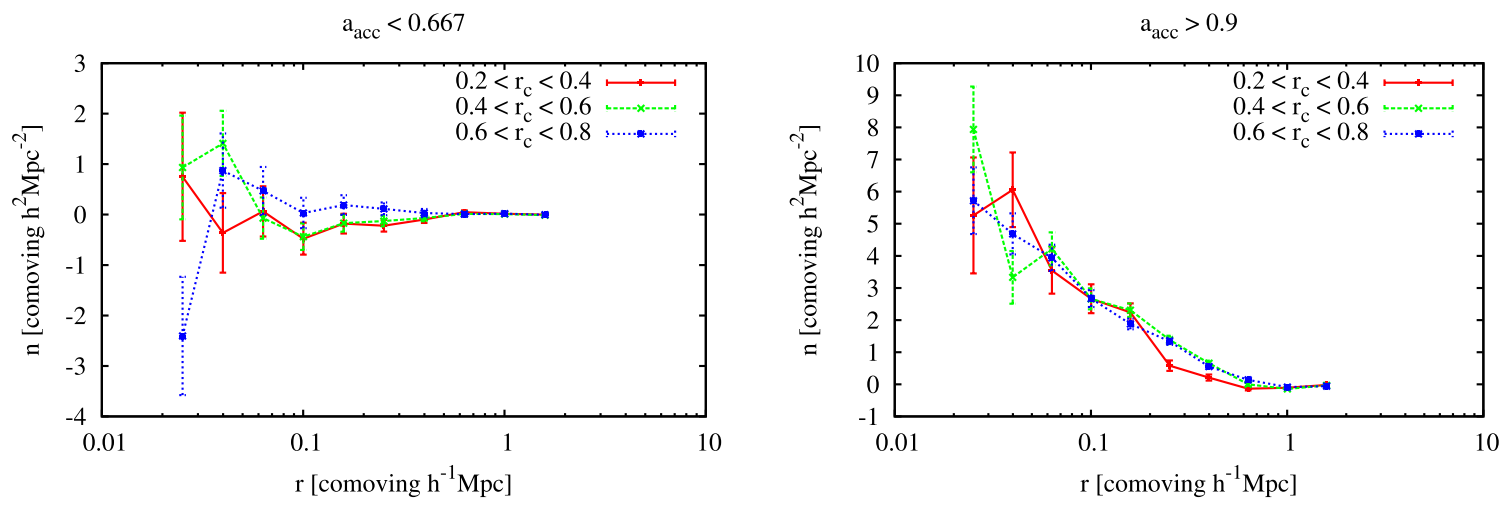

Figure A1. Subhalo correlations in a simulation 64 times bigger than that used in Chamberlain et al. (2015). The left-hand panel shows the measured correlations for subhaloes accreted $5 \mathrm{Gyr}$ ago. Thus, these subhaloes have been in their hosts longer than a dynamical time. From this, we conclude that there are no correlations outside $r_{\text {tidal }}$ after a dynamical time. The right-hand panel shows the correlations for subhaloes accreted more recently, which are consistent with no tidal stripping.

This paper has been typeset from a $\mathrm{T}_{\mathrm{E}} \mathrm{X} / \mathrm{LT} \mathrm{E} \mathrm{X}$ file prepared by the author. 